Gut and Liver, Vol. 10, No. 1, January 2016, pp. 101-108

\title{
Doxazosin Treatment Attenuates Carbon Tetrachloride-Induced Liver Fibrosis in Hamsters through a Decrease in Transforming Growth Factor $\beta$ Secretion
}

Martin Humberto Muñoz-Ortega*, Raúl Wiliberto Llamas-Ramírez ${ }^{\dagger}$, Norma Isabel Romero-Delgadillo ${ }^{\dagger}$, Tania Guadalupe Elías-Flores ${ }^{\dagger}$, Edgar de Jesus Tavares-Rodríguez ${ }^{\dagger}$, María del Rosario Campos-Esparza ${ }^{\dagger}$, Daniel Cervantes-García*, Luis Muñoz-Fernández $z^{\ddagger}$, Martin Gerardo-Rodríguez ${ }^{\S}$, and Javier Ventura-Juárez ${ }^{\dagger}$

Departments of *Chemistry, ${ }^{\dagger}$ Morphology, Center of Basic Sciences, Autonomous University of Aguascalientes, ${ }^{\ddagger}$ Department of Pathology, Centenary Hospital Miguel Hidalgo, and ${ }^{s}$ Department of Physiology, Center of Basic Sciences, Autonomous University of Aguascalientes, Aguascalientes, Mexico

See editorial on page 10.

Background/Aims: The development of therapeutic strategies for the treatment of cirrhosis has become an important focus for basic and clinical researchers. Adrenergic receptor antagonists have been evaluated as antifibrotic drugs in rodent models of carbon tetrachloride (CCl4)-induced cirrhosis. The aim of the present study was to evaluate the effects of carvedilol and doxazosin on fibrosis/cirrhosis in a hamster animal model. Methods: Cirrhotic-induced hamsters were treated by daily administration of carvedilol and doxazosin for 6 weeks. Hepatic function and histological evaluation were conducted by measuring biochemical markers, including total bilirubin, aspartate aminotransferase, alanine aminotransferase and albumin, and liver tissue slices. Additionally, transforming growth factor $\beta$ (TGF- $\beta$ ) immunohistochemistry was analyzed. Results: Biochemical markers revealed that hepatic function was restored after treatment with doxazosin and carvedilol. Histological evaluation showed a decrease in collagen type I deposits and TGF- $\beta$-secreting cells. Conclusions: Taken together, these results suggest that the decrease in collagen type I following treatment with doxazosin or carvedilol is achieved by decreasing the profibrotic activities of TGF- $\beta$ via the blockage of $\alpha 1$ - and $\beta$-adrenergic receptor. Consequently, a diminution of fibrotic tissue in the CCl4-induced model of cirrhosis is achieved. (Gut Liver 2016;10:101-108)

Key Words: Cirrhosis; Adrenergic pathway; Adrenergic recep- tor antagonist; Collagen; Transforming growth factor beta

\section{INTRODUCTION}

In 2010, cirrhosis caused for alcohol consumption was responsible for 493,300 deaths worldwide. In the same year, liver cirrhosis occupied the fourth place of fatalities in Mexico with 28,369 deaths. Liver fibrosis (LF) and cirrhosis are the result of a chronic process of inflammation and tissue repair, with accumulation of connective tissue. ${ }^{1,2}$ The deposition of collagen is caused by an important imbalance between production and degradation of the extracellular matrix, even if origin of the insult is viral (hepatitis B or C virus), an autoimmune/metabolic disease (autoimmune hepatitis or hemochromatosis), or the consumption of drugs and/or alcohol. ${ }^{3,4}$ When damage is persistent, the hepatic architecture is altered with fibrotic bands, regenerative nodules and vascular distortion. In chronic liver disease, prolonged and repeated activation of hepatic stellate cells (HSCs) produces LF, as characterized by widespread scar formation and perturbation of liver function and architecture. ${ }^{2,4}$

At the initiation of the fibrotic process, HSCs show fibrogenic and contractile activities in response to platelet-derived growth factor $\beta$, transforming growth factor $\beta$ (TGF- $\beta$ ), and epidermal growth factor. ${ }^{4,5}$ Later, activated HSCs increase the deposition of extracellular matrix proteins, like elastin, fibrillar collagen I, II, and V, nonfibrillar collagen IV and VI. In the stage of resolution, the inflammatory process is diminished, and activated HSCs can reduce their activation by acquiring a quiescent phenotype or cleared by apoptosis. ${ }^{4,6}$

Some drugs and pathways are being used as target for the

Correspondence to: Javier Ventura-Juárez

Department of Morphology, Center of Basic Sciences, Autonomous University of Aguascalientes, Av. Universidad No. 940, Ciudad Universitaria, Aguascalientes 20131, Mexico

Tel: +52-01449-9-108425, Fax: +52-01449-9-108425, E-mail: jventur@correo.uaa.mx

Received on November 1, 2014. Revised on January 10, 2015. Accepted on February 2, 2015. Published online November 18, 2015 pISSN 1976-2283 eISSN 2005-1212 http://dx.doi.org/10.5009/gnl14459

@) This is an Open Access article distributed under the terms of the Creative Commons Attribution Non-Commercial License (http://creativecommons.org/licenses/by-nc/4.0) which permits unrestricted non-commercial use, distribution, and reproduction in any medium, provided the original work is properly cited. 
blockage of fibrotic process, like angiogenesis inhibitors, antihypertensive drugs like angiotensin inhibitors, downregulation of TGF- $\beta$, and cell therapy strategies. ${ }^{7}$

HSCs have adrenergic receptors (ARs) that mediate their metabolic, proliferative and regenerative activities. The activation of ARs initiates profibrotic changes in HSCs. Also, $\alpha$ - and $\beta$-AR antagonists decrease portal pressure. $\beta$-AR antagonists mediate the reduction of portal venous blood flow by a nonselective effect, while the $\alpha_{1}$-AR antagonist reduces the intrahepatic resistance. ${ }^{8}$ Oben et al. ${ }^{9}$ demonstrated that cultured mice HSCs activated with isoprenaline, a $\beta$-AR agonist, had an increment of $\alpha$-smooth muscle actin-positive HSCs. Recent reports have shown that hepatic damage is reduced in mice models through the use of prazosin, an antagonist of the AR $\alpha_{1}$, or via a chemical sympathectomy induced by 6-hydroxidopamine (6OHDA). ${ }^{10,11}$

Carvedilol is a $\beta$-AR antagonist used as an antihypertensive drug. It is used clinically to reduce portal hypertension in patients with liver damage. ${ }^{12}$ Due to its antioxidant and antifibrotic effects, it has been employed for diminishing LF when is coadministered with carbon tetrachloride $(\mathrm{CCl} 4)$ in rats before cirrhosis has been established. ${ }^{13,14}$ On the other hand, doxazosin has hypotensive activity in arteries through the inhibition of $\alpha_{1}$ AR. In addition, doxazosin is used for the treatment of benign prostatic hyperplasia and arterial hypertension due induction of prostate epithelial and smooth muscle cell apoptosis. ${ }^{15}$ Besides, when overexpression of TGF- $\beta$ is inhibited, hepatic fibrosis is reduced, for this reason inhibitors of TGF- $\beta$ are currently explored as potential drugs. ${ }^{16}$

The aim of the present study was to chemically inhibit the sympathetic pathway of $\alpha_{1}$-AR with doxazosin in order to determine whether this drug can attenuate the development of CCl4-induced LF in a hamster model of cirrhosis.

\section{MATERIALS AND METHODS}

\section{Animal model of cirrhosis}

Golden male hamsters (Mesocricetus auratus) from 6- to 8-week-old (weight, 100 to $160 \mathrm{~g}$ ), were maintained on a light/ dark cycle (12:12). Food (Purina Rat Chow from Ralston Purina Company, St. Louis, MO, USA) and water were provided ad libitum in the animal facility of the Autonomous University of Aguascalientes. The protocol of all experiments had been approved by the Institutional Bioethical Committee.

The following four groups were formed to establish the model of experimental cirrhosis: untreated control group $(n=15)$; vehicle group administered with sodium petrolatum by intraperitoneal injection $(n=15)$; and two groups in which cirrhosis was induced by intraperitoneal injection of $\mathrm{CCl} 450 \mathrm{mg} / \mathrm{kg}(\mathrm{n}=15)$ or $100 \mathrm{mg} / \mathrm{kg}(\mathrm{n}=15)$ twice weekly. Five animals of each group were euthanized at 8,12 , and 16 weeks to analyze the development of cirrhosis. Survival rate was evaluated at 16 weeks.

\section{Antifibrotic treatment}

To test the pharmacological effect of doxazosin and carvedilol, both drugs were administered for 6 weeks to hamsters once cirrhosis was induced with CCl4 $50 \mathrm{mg} / \mathrm{kg}$. Animals were divided into five groups: (1) untreated control hamsters ( $\mathrm{n}=5),(2)$ placebo $(0.2 \mathrm{~mL}$ of saline solution administered orally during 6 weeks; $n=5)$, (3) doxazosin (1.0 mg/kg of Cardura ${ }^{\circledR}$; Pfizer, New York, NY, USA, daily by oral administration during 6 weeks; $\mathrm{n}=5)$, (4) carvedilol (1.2 mg/kg of Dilatred ${ }^{\circledR}$; Roche, Basel, Switzerland, daily by oral administration; $n=5$ ), and (5) 6-OHDA (30 mg/kg; Sigma-Aldrich, St. Louis, MO, USA, by intraperitoneal injection twice weekly; $n=5$ ).

\section{Histological techniques}

Liver biopsies were obtained from euthanized animals. Tissues were histologically processed for Masson. To study the differential deposition of collagen type I and III, Sirius Red staining were performed. ${ }^{17}$ Histopathological comparison was made between experimental groups and representative photographs from three histological preparations of human with liver cirrhosis obtained from the Centenario Hospital Miguel Hidalgo, Aguascalientes, Mexico, according to the regulative guidelines. After treatment with carvedilol and doxazosin, preparations with the Sirius red technique were compared by measurement of fibrotic area with a Carl Zeiss Axioscop 40 coupled with Image Pro Plus V4.5.1 software and Cool Snap-Pro Color camera (Media Cybernetics, Rockville, MD, USA).

\section{Liver function tests}

Whole blood samples (1.5 to $2.0 \mathrm{~mL}$ ) were centrifuged, and serum was stored at $-20^{\circ} \mathrm{C}$ until use. Biochemical markers (all obtained from Spinreact, Girona, Spain) of hepatic function, total bilirubin (TB), aspartate aminotransferase (AST), alanine aminotransferase (ALT), and albumin were measured employing a spectrophotometric semiautomatic bts-350 analyzer (Biosystems, Quezon City, Philippines).

\section{TGF- $\beta$ immunohistochemistry}

To evaluate secreting TGF- $\beta$ cellular population, liver tissue slides were incubated with a rabbit antihuman TGF- $\beta$ (Media Cybernetics), for 12 hours at $4^{\circ} \mathrm{C}$. As secondary antibody a goat antipolyvalent Biotin-Streptavidin HRP (Thermo Scientific, Waltham, MA, USA) was used. The peroxidase activity was developed with diaminobenzidine. Densities of TGF- $\beta$ positive cells were counted as cells $/ \mathrm{mm}^{2}$.

\section{Statistical analysis}

GraphPad Prism V5 software was employed for statistical analysis. Data are expressed as the mean \pm standard deviation of five animals. Significant differences between mean values were evaluated by using the two-way analysis of variance test with the 
Tukey posthoc. Statistical significance was considered at $\mathrm{p}<0.05$.

\section{RESULTS}

\section{CCl4-induced fibrosis in hamsters}

Hamsters were treated by intraperitoneal injections of $\mathrm{CCl} 4$ for 8,12 , and 16 weeks, and survival was evaluated every week. After 16 weeks, survival in the $50 \mathrm{CCl} 4$ group was $70 \%$ and in the $100 \mathrm{CCl} 4$ group 50\%. Deaths were not observed in the control or vehicle groups (Fig. 1A).

The increase in plasmatic levels of AST and ALT was observed at 8,12 , and 16 weeks of $\mathrm{CCl} 4$ treatment. With both doses of $\mathrm{CCl} 4,50$ and $100 \mathrm{mg} / \mathrm{kg}$, significant increases in AST and ALT were observed at 16 weeks (Fig. 1B). Both hepatic enzymes are biochemical indicators for liver damaged induced with $\mathrm{CCl} 4$. Plasmatic levels of albumin showed a marked decrease after treatment for 12 and 16 weeks. These results reveal the development of ascites and reduction of capacity of liver metabolism, which are the major complications of liver cirrhosis and portal hypertension (Fig. 1C). Determination of TB showed an increase at 12 and 16 weeks, which demonstrated advanced liver damage with biliary cholestasis (Fig. 1D).

After 8 weeks, Masson histological analysis showed organized and structured hepatic tissue with normal stromal collagen in control and vehicle groups. In the same groups, Sirius red-stained liver sections revealed the presence of collagen III (in green) surrounding the portal system. The 50CCl4 and $100 \mathrm{CCl} 4$ groups showed abnormal and coarse blocks of collagen (which form septa at the interlobular level), laminar fibrosis and periportal fibrosis with abundant collagen type III (Fig. 2A and B). Histological analysis at 12 weeks of $\mathrm{CCl} 4$-induction revealed normal conformation of hepatic lobules and portal vein in control and vehicle groups. At the same time, the 50CCl4 and $100 \mathrm{CCl} 4$ had coarse blocks of collagen type III forming abnormal septa and regenerative nodules. At 16 weeks of treatment, in the $50 \mathrm{CCl} 4$ and $100 \mathrm{CCl} 4$ groups the Masson stain
A
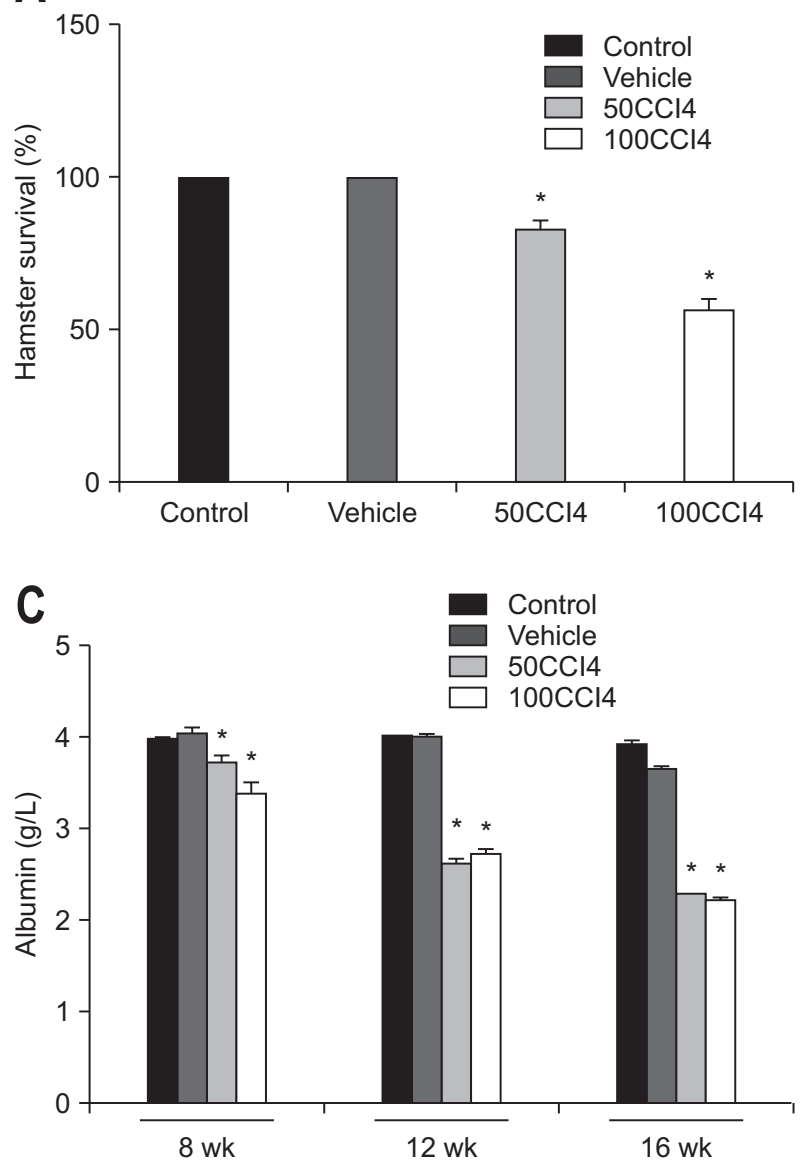

B

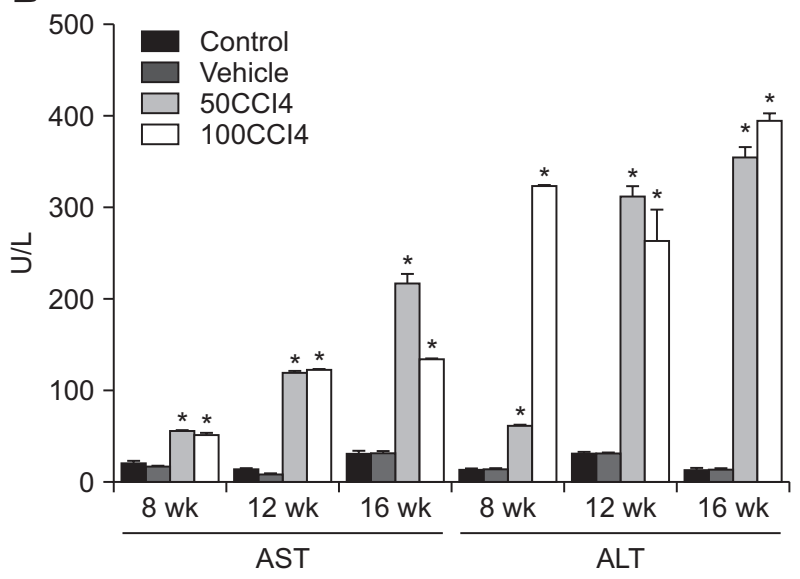

D

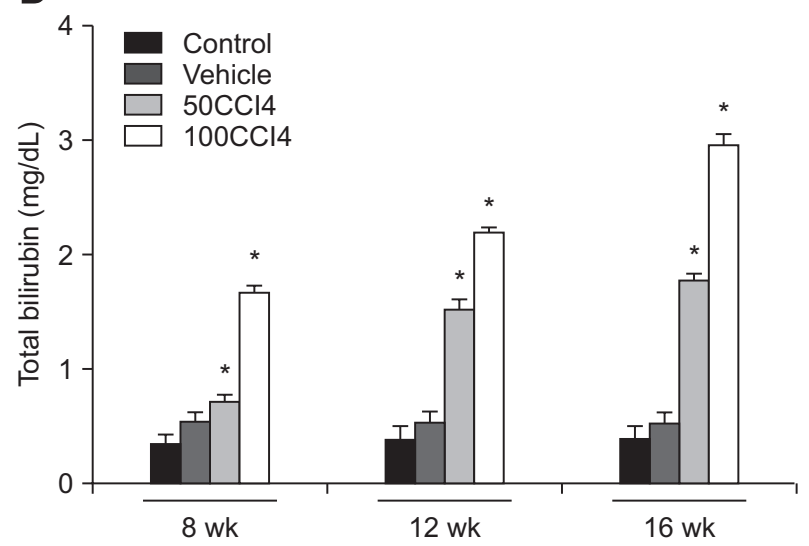

Fig. 1. Kinetics of biochemical markers during the induction of hepatic damage with carbon tetrachloride (CCl4). (A) In contrast to the 100\% survival in the control group, 70\% and 50\% survival was observed in the 50CCl4 and 100CCl4 groups, respectively. (B) An increase in the plasmatic activity of alanine aminotransferase (ALT) and aspartate aminotransferase (AST) was observed during treatment with CCl4 (at both doses). (C) A significant decrease in plasmatic albumin was detected at 12 and 16 weeks in both the 50CCl4 and 100CCl4 animals compared with the control. (D) A significant increase in total bilirubin was found in CCl4-treated animals, which revealed a cirrhotic process. Values are represented as the mean \pm SD. ${ }^{*} \mathrm{p}<0.05$. 
A
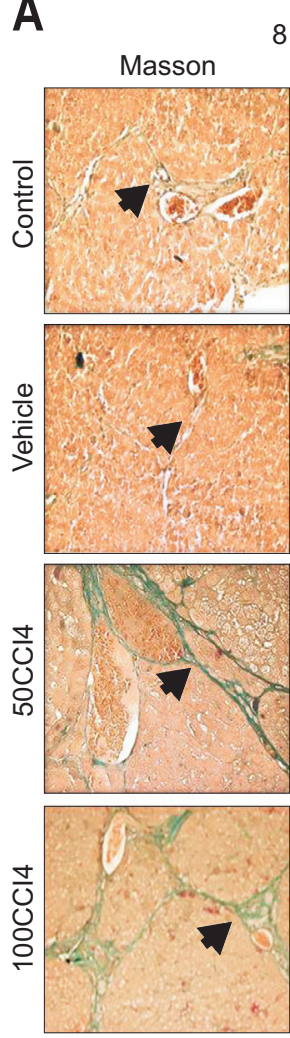

8 wk

Sirius red
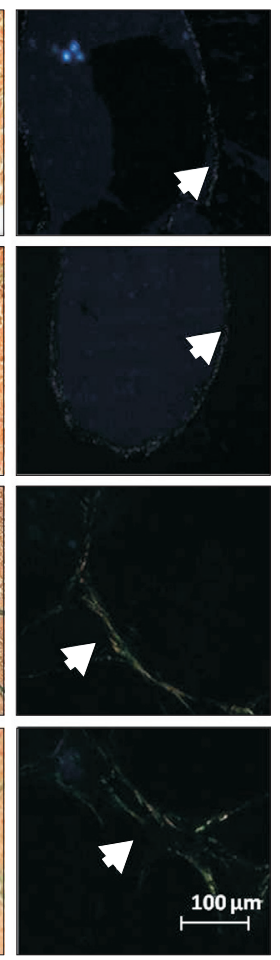

B
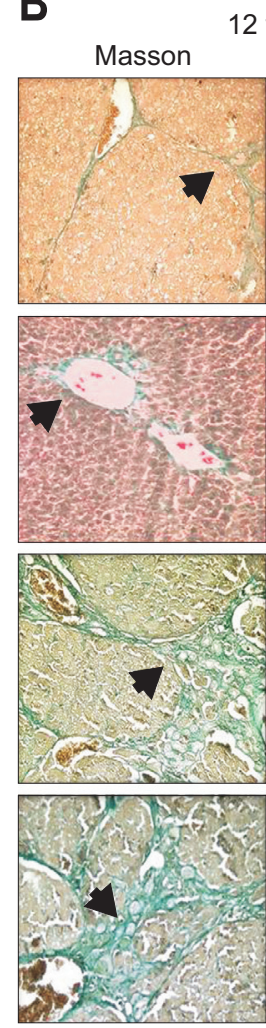

2 wk
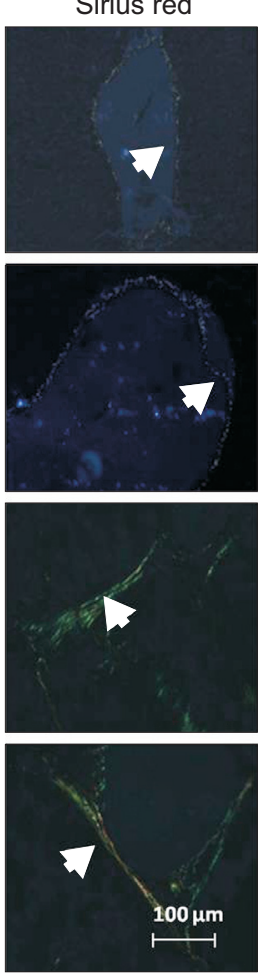

C
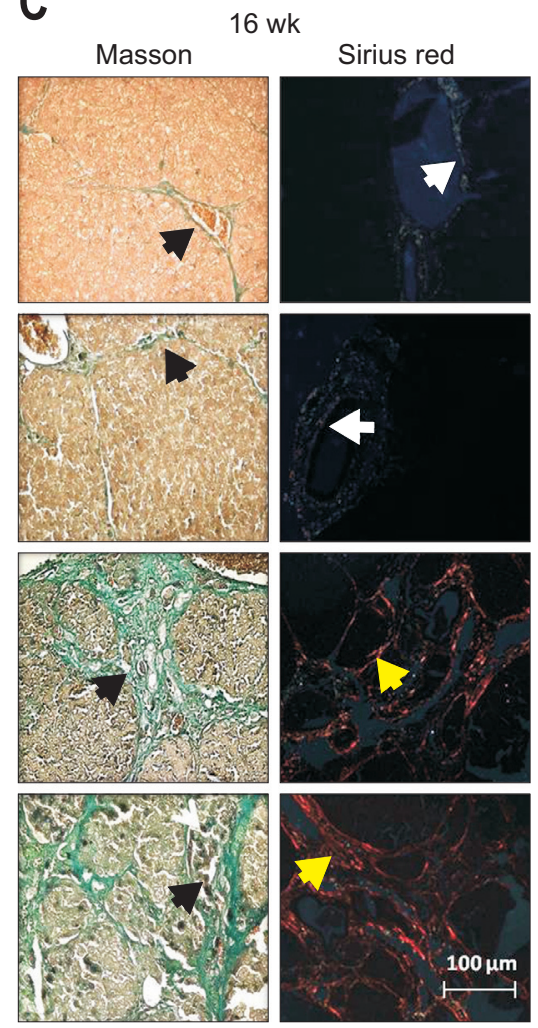

D
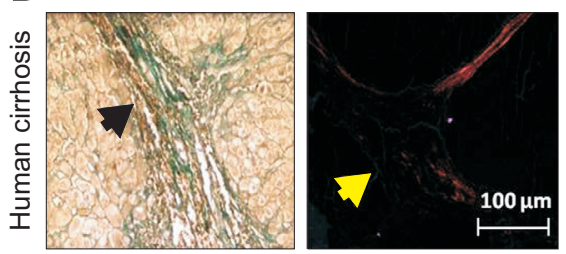

Fig. 2. Evaluation of collagen type I in the $50 \mathrm{CCl} 4$ and $100 \mathrm{CCl} 4$ groups at 8,12 , and 16 weeks of induction. At 8 and 12 weeks, liver slides from the animals given $50 \mathrm{CCl} 4$ (A) and 100CCl4 (B) showed an increase in deposits of collagen (black arrows), which mainly consisted of collagen type III (white arrows) with interlobular septa and periportal fibrosis. (C) After 16 weeks of $\mathrm{CCl} 4$ treatment, liver sections presented gross blocks of collagen type I and small regenerative nodules. (D) Deposits of collagen type I (yellow arrows) and regenerative nodules in animals given 50CCl4 and $100 \mathrm{CCl} 4$ resembled the Masson and Sirius red staining profile from human cirrhotic livers.

$\mathrm{CCl} 4$, carbon tetrachloride.

showed coarse collagen deposits which form several regenerative nodules. The Sirius red stain demonstrated the deposition of collagen type I (in red), which appeared surrounding smaller regenerative nodules, these data demonstrated a more advanced injury than 12 weeks (Fig. 2C). On the other hand, the histopathological comparison between the livers from CCl4-induced fibrosis in hamsters at 16 weeks and human cirrhotic liver samples obtained from patients revealed similar nodules surrounded by fibers of collagen type I (Fig. 2D).

Due to the 70\% survival and biochemical evidence of cirrhosis in the 50CCl4 group, we selected this hamster model to test doxazosin and carvedilol, and evaluate their antifibrotic effect and the possible diminution of the advanced phases of liver disease.

\section{Diminution of fibrosis with the $\alpha_{1}$ - and $\beta$-AR antagonist}

The plasma levels of AST, ALT, bilirubin and albumin, were measured to determine liver function in cirrhotic hamsters treated with 6-OHDA, doxazosin or carvedilol. The results for each compound are in accordance with the histological data of liver function for the same groups.

With 6-OHDA, doxazosin and carvedilol, we observed a decrease in the biochemical markers of liver tissue damage, including AST, ALT and TB. There was no significant difference in the albumin levels between the placebo and 50CCl4 groups. However, a significant increase in the plasmatic level of albumin was found with 6-OHDA, doxazosin and carvedilol treatments when compared with the control group. Compared with the placebo group, these biochemical markers did not change significantly 
A

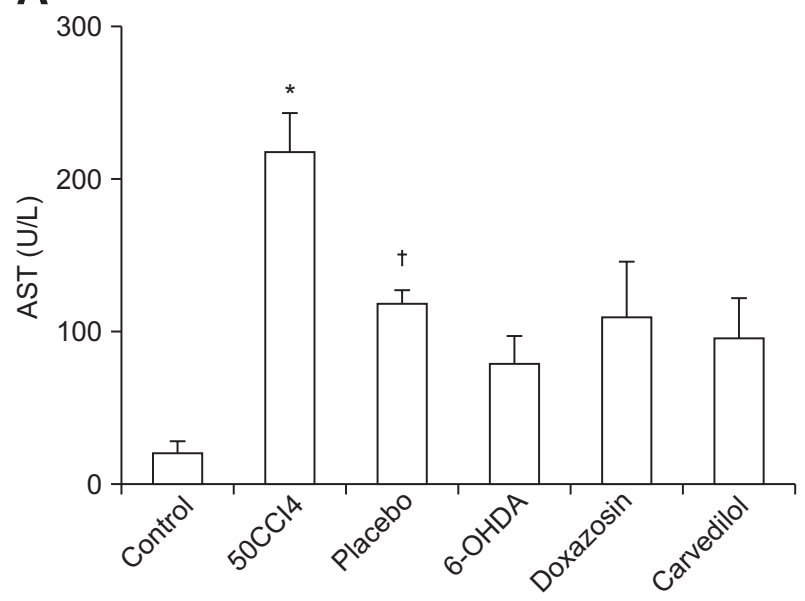

C

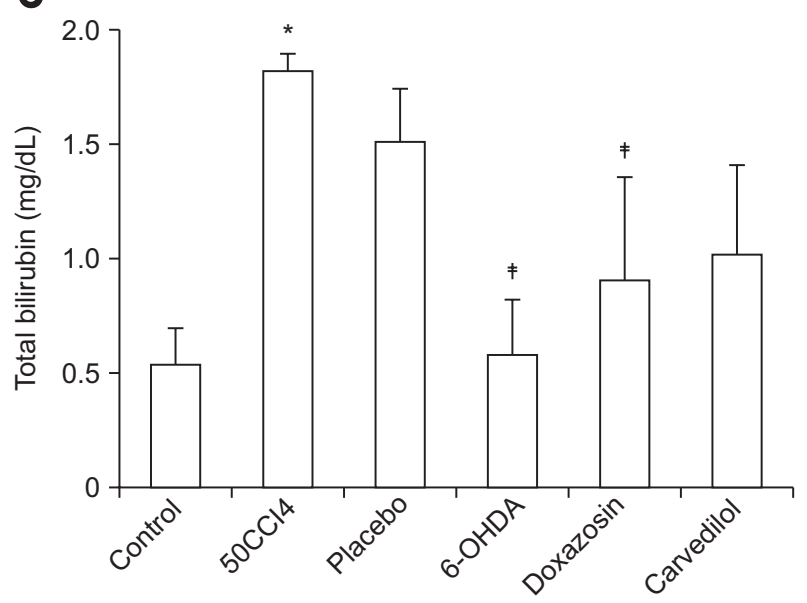

B

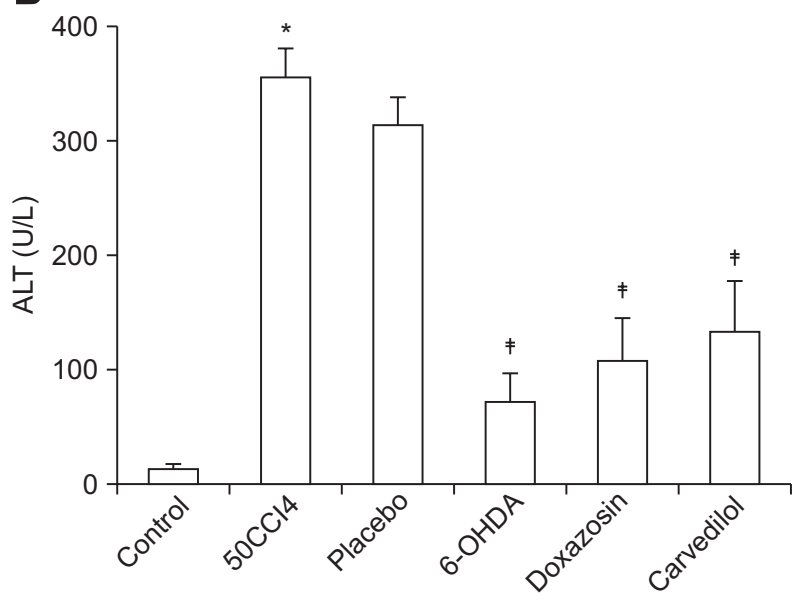

D

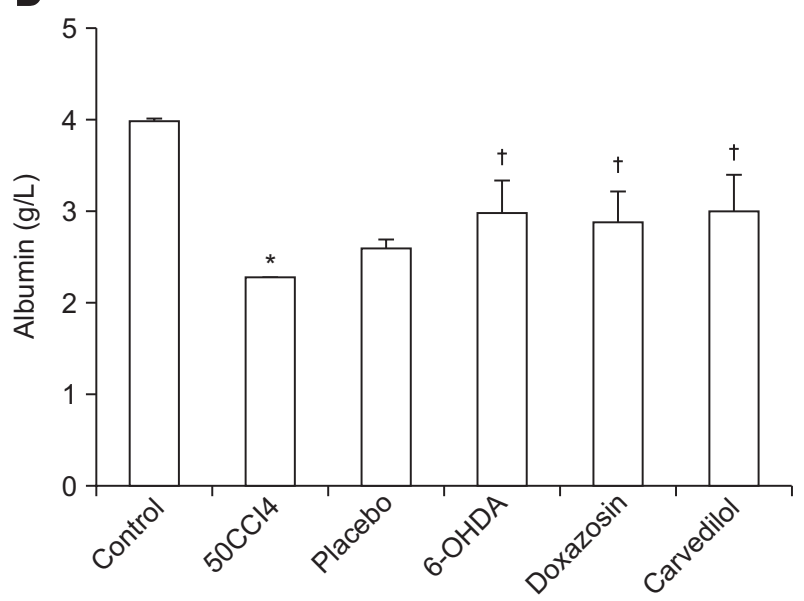

Fig. 3. Recovery of hepatic function in hamsters after carbon tetrachloride (CCl4)-induced fibrosis and treatment with adrenergic receptor (AR) antagonists. (A) There was no significant difference in plasmatic levels of aspartate aminotransferase (AST) in hamsters treated or not treated with AR antagonists. However, a significant reduction in alanine aminotransferase (ALT) (B) and total bilirubin (C) was achieved after treatment with 6-hydroxydopamine (6-OHDA), doxazosin, or carvedilol. (D) Albumin synthesis demonstrated restitution of hepatic function after treatment with AR antagonists, thus corroborating the same effect previously demonstrated with histological data. Values are represented as the mean \pm SD. ${ }^{*} \mathrm{p}<0.05$ versus control; ${ }^{\dagger} \mathrm{p}<0.05$ versus $50 \mathrm{CCl} 4 ;{ }^{\ddagger} \mathrm{p}<0.05$ versus placebo.

after a chemical sympathectomy with 6-OHDA (Fig. 3).

Based on analysis of liver slides, we evaluated the possible diminution of fibrosis in the groups treated with 6-OHDA, doxazosin or carvedilol. To evaluate if there was difference in collagen deposition we performed Masson's trichrome staining in experimental groups, we observed diminution of total collagen in groups treated with 6-OHDA, doxazosin and carvedilol. The measurement of the fibrotic area clearly demonstrated a three-fold reduction in collagen deposits after treatment with $\alpha_{1}$ - and $\beta$-AR antagonists. The ratio of the fibrotic area to the total liver area was significantly reduced in the doxazosin group (3.35\% $\pm 2.29 \%)$ compared with the placebo group $(9.85 \% \pm 4.51 \%)$. No significant difference was observed in the fibrotic area of the doxazosin, 6-OHDA and carvedilol treated groups (Fig. 4A and B). After, liver slides were analyzed with Sirius red staining and visualized under polarized light, as previously described. In the group with cirrhosis induced by $50 \mathrm{CCl} 4$, we observed the presence of collagen type I in the liver parenchyma. After 6 weeks of placebo administration, the presence of collagen type I persisted, with no evidence of changes in the fibrotic area when compared with $50 \mathrm{CCl} 4$ group. The 6-OHDA group showed a marked decrease in accumulated collagen type I in the fibrotic area. Moreover, after this treatment we observed expression of collagen type III and parenchyma restructuring. Treatment with the $\alpha_{1}$-AR antagonist, doxazosin, promoted the diminution of liver cirrhosis, including a decrease in collagen type I deposits, enlargement of regenerative nodules, and the presence of thin and incomplete septa. Otherwise, we observed that the presence of collagen type III was similar to the control group, after 6 weeks of doxazosin administration. At the same time, in hamsters treated with carvedilol, the septa and regenerative nodules were similar to those observed with doxazosin treatment, while fibrotic tissue was reduced (Fig. 4C). These results suggest that treatment with doxazosin may reduce fibrosis 
A
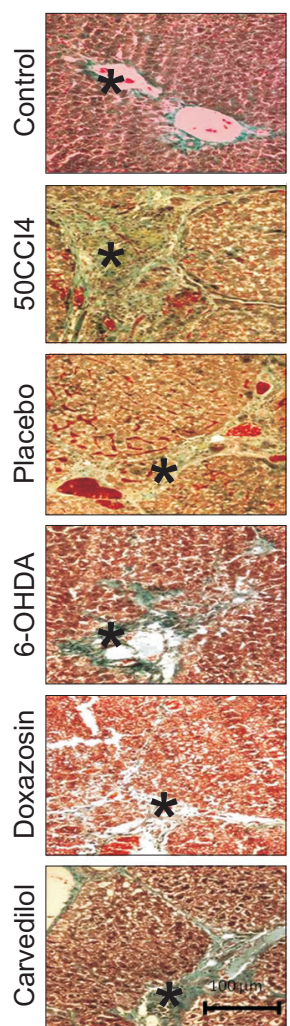
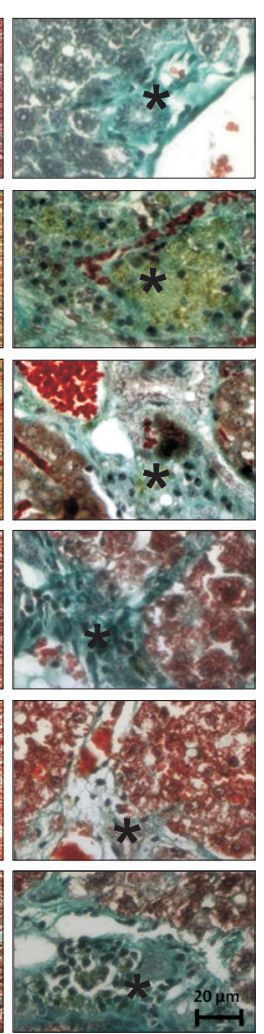

B

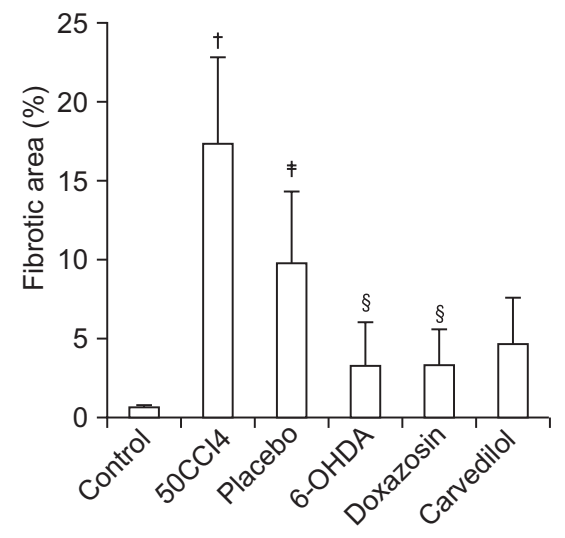

C

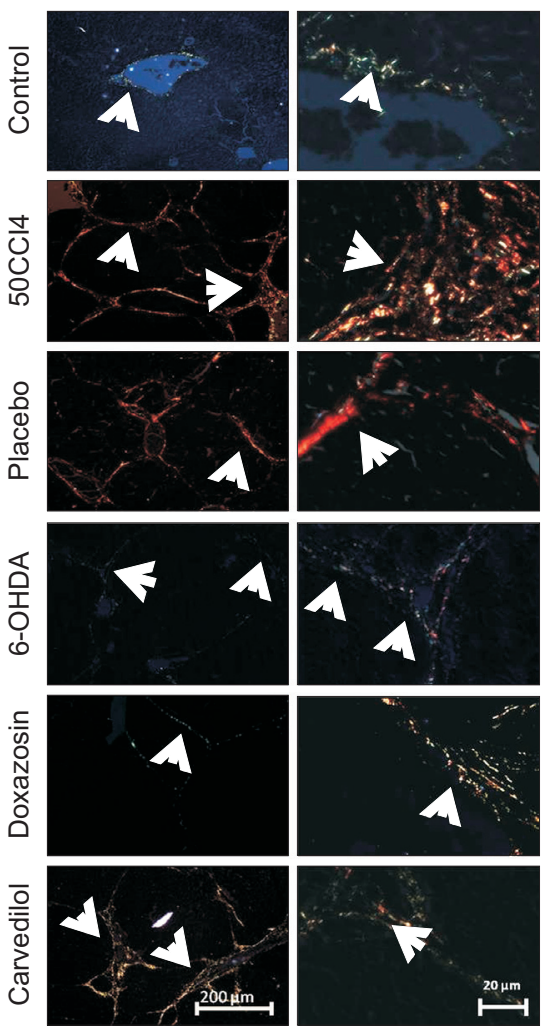

Fig. 4. Sirius red staining under polarized light demonstrates the antifibrotic effects of doxazosin and carvedilol on carbon tetrachloride (CCl4)-induced fibrosis. (A) Masson trichrome staining revealed an increase in collagen deposition (*) in the portal tract in the 50CCl4 and placebo groups. However, an attenuation of collagen deposition was observed in the 6-hydroxydopamine (6-OHDA), doxazosin and carvedilol groups. (B) A significant reduction in the fibrotic area was observed in doxazosin- and carvedilol-treated animals. (C) The control group showed normal histological architecture with collagen type III. In the liver sections of hamsters treated with $50 \mathrm{mg} / \mathrm{kg}$ of CCl4, there were multiple regenerative nodules (*) surrounded by collagen type I. The placebo group had a reduction of collagen deposits (white arrows) with a normal level of collagen type I. The chemical sympathectomy generated incomplete septa of collagen type III (white arrows), forming enlarged regenerative nodules (*). Doxazosin treatment promoted a shift to collagen type III (white arrows); no evidence of regenerative nodules was observed. Carvedilol treatment resulted in little collagen type III (white arrows) and few regenerative nodules $(*)$. Values are represented as the mean \pm SD. ${ }^{\dagger} p<0.05$ versus control; ${ }^{\ddagger} p<0.05$ versus $50 \mathrm{CCl} 4 ;{ }^{8} \mathrm{p}<0.05$ versus placebo.

after hepatic damage induced with $\mathrm{CCl} 4$, and may even restore hepatic function.

Since activated HSCs overexpress TGF- $\beta$ in response to liver injury, we tested whether the presence of this profibrotic cytokine is associated with the reduction of LF. The doxazosin treatment significantly decreased the number of TGF- $\beta$-positive cells $\left(17.33 \pm 3.21\right.$ cells $\left./ \mathrm{mm}^{2}\right)$ compared with the placebo group $\left(100.7 \pm 10.07\right.$ cells $\left./ \mathrm{mm}^{2}\right)$ (Fig. 5). There were notably fewer TGF- $\beta$ secreting cells in the doxazosin than carvedilol group. Overall, these data indicate that the doxazosin treatment possesses an antifibrotic effect and promotes the recovery of hepatic function in the presence of cirrhosis.

\section{DISCUSSION}

The current contribution explores a new application of doxazosin, an antihypertensive drug, as a blocker of the sympathetic pathway of $\alpha_{1}$-ARs in liver cirrhotic. We demonstrate that treatment with doxazosin reduced collagen type I deposits by changing them to collagen type III, and decreased TGF- $\beta$ secreting cells in the hepatic parenchyma of hamsters with cirrhosis induced by $\mathrm{CCl} 4$.

LF is the final step of chronic inflammatory liver diseases and is characterized by HSC proliferation and differentiation to myofibroblast-like cells, which deposit collagen type I in extracellular matrix. ${ }^{4}$ Studies of chronic liver diseases, like cirrhosis, have been carried out in a variety of in vitro and in vivo models. ${ }^{18,19}$ An animal model of CCl4-induced hepatic fibrosis in hamsters has been employed to study the attenuation of fibrosis by the blockade of angiotensin activation. ${ }^{20}$

In the CCl4-induced hepatic fibrosis hamster model, plasmatic levels of TB, ALT, and AST were increased, while albumin was decreased. These biochemical changes, found at 8, 12, and 16 weeks, are similar to those observed with human cirrhosis and 
A

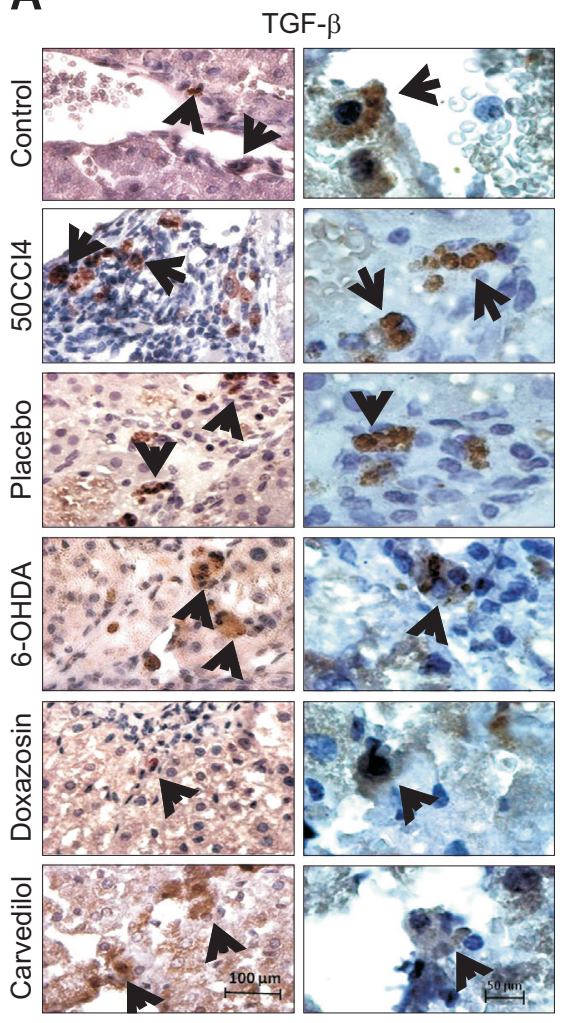

B

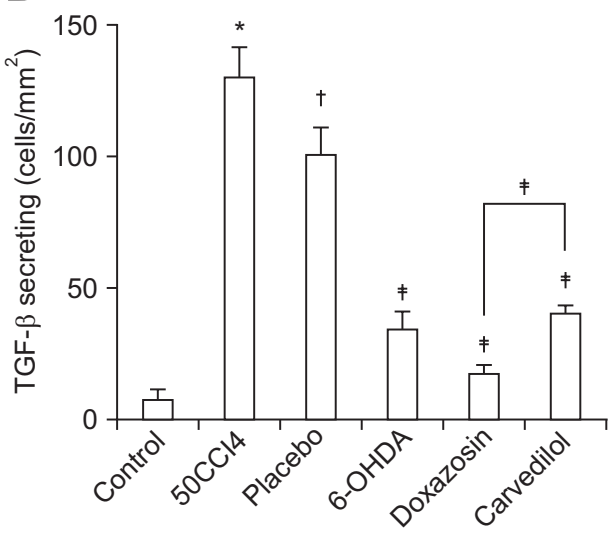

Fig. 5. Doxazosin decreased the transforming growth factor $\beta$ (TGF- $\beta$ )-secreting cells. (A) Representative histological sections of liver for immunohistochemical evaluation of TGF- $\beta$ secreting cells; magnifications at left and right columns are $10 \times$ and $40 \times$, respectively. (B) TGF- $\beta$ secreting cells were significantly reduced in cirrhotic hamsters after doxazosin treatment. Values are represented as the mean \pm SD.

CCl4, carbon tetrachloride; 6-OHDA, 6-hydroxydopamine. ${ }^{*} \mathrm{p}<0.05$ versus control; ${ }^{\dagger} \mathrm{p}<0.05$ versus $50 \mathrm{CCl} 4 ;{ }^{\ddagger} \mathrm{p}<0.05$ versus placebo.

liver failure. ${ }^{21,22}$ Both the decrease in albumin and the increase in transaminases corroborate hepatic damage.

Histological data from control animals show a normal architecture of hepatocytes arranged in sinusoids, without evidence of fibrosis. Contrarily, in the $50 \mathrm{CCl} 4$ and $100 \mathrm{CCl} 4$ groups the hepatic architecture underwent a distortion, in which the regenerative nodules were surrounded by collagen type I septa. Once the CCl4-induced cirrhosis was established, we explored the possible therapeutic effect of doxazosin or carvedilol treatment to revert this condition.

Doxazosin has not yet been tested as an antifibrotic drug in liver cirrhotic animal models or in patients. Doxazosin treatment induce a reduction in renal fibrosis and myocardial interstitial fibrosis, ${ }^{23-25}$ and due its role in the reduction of smooth muscle tone, it has been used in the treatment of benign prostatic hyperplasia. ${ }^{15,23}$ Since HSCs are the main fibrogenic cells in liver and express $\alpha_{1}-A R,{ }^{9,10}$ we examined the possible inhibitory effect of doxazosin treatment on collagen deposition in the pathogenesis of cirrhosis. Doxazosin effectively reduced the content of collagen type I, and achieved the recovery of hepatic function after liver injury. We argue that this antifibrotic effect owes itself to the selective inhibition of $\alpha_{1}$-ARs in HSCs, which would then decrease collagen synthesis. HSCs and Kupffer cells possess the $\alpha_{1}$-AR. Evidence of increment of extracellular matrix with collagen deposition has been reported after noradrenaline administration in cultured HSCs, leading to the activation of intracellular kinases, like pI3K and MEK which are implicated in cell proliferation and survival. Also, HSCs and Kupffer cells are involved in the production of TGF- $\beta$ in the liver, and when the $\alpha_{1}-\mathrm{AR}$ is blockaded, their proliferation rate is reduced and finally the secretion of TGF- $\beta$ diminished. ${ }^{9,10}$

On the other hand, Hamdy and El-Demerdash ${ }^{14}$ reported the antifibrotic activity of carvedilol when it was coadministered with $\mathrm{CCl} 4$ for 6 and 8 weeks. In accordance with this report, the present results clearly demonstrate that carvedilol possesses antifibrotic activity once cirrhosis is established, evidenced by the fact that collagen type I deposits were reverted after 6 weeks of carvedilol treatment.

Furthermore, in cirrhotic hamsters treated with doxazosin, lowered levels of TGF- $\beta$ promoted a decrease in fibrotic tissue, probably by a reduction in the production of extracellular matrix proteins, as previously reported. ${ }^{26}$ Similar results have been observed in cirrhotic rats $\mathrm{CCl} 4$-induced after treatment with prazosin; ${ }^{11}$ fibrotic area was reduced by $83 \%$, accompanied by a reduction in the levels of collagen type I mRNA and tissue inhibitor of matrix metalloproteinases-1 mRNA. Aldaba-Muruato et $a{ }^{27}{ }^{27}$ demonstrated that TGF- $\beta$ synthesis and collagen type I deposition were significantly decreased after treatment with allopurinol, which is a candidate drug for the treatment of liver diseases in human patients.

In conclusion, the current results suggest that when cirrhosis CCl4-induced hamsters are treated with doxazosin to blockage the $\alpha_{1}$-AR on HSCs, a visible reduction in deposits of collagen type I are observed. This effect of doxazosin could be related to 
a decrease in TGF- $\beta$ synthesis. Hence, the blockage of $\alpha_{1}$-ARs with doxazosin may be a useful therapeutic strategy for treatment of cirrhotic patients.

\section{CONFLICTS OF INTEREST}

No potential conflict of interest relevant to this article was reported.

\section{ACKNOWLEDGEMENTS}

The authors of this study would like to thank Liseth R. AldabaMuruato for her guidance and technical assistance, and Bruce Allan Larsen in editing the use of English language.

M.H.M.O. and R.W.L.R. were recipients of a fellowship from CONACyT. This work was funded by CONACyT-SEP 134487, CONACyT-SEP 241312 and UAA-PIBB 12-3 grants.

\section{REFERENCES}

1. Ueha S, Shand FH, Matsushima K. Cellular and molecular mechanisms of chronic inflammation-associated organ fibrosis. Front Immunol 2012;3:71.

2. Firpi RJ, Clark V, Soldevila-Pico C, et al. The natural history of hepatitis C cirrhosis after liver transplantation. Liver Transpl 2009; 15:1063-1071.

3. Aghemo A, Colombo M. Cirrhosis regression in chronic hepatitis C: an old tale with a new ending. Gastroenterology 2009;136:14471449.

4. Wynn TA. Cellular and molecular mechanisms of fibrosis. J Pathol 2008;214:199-210.

5. Holt AP, Salmon M, Buckley CD, Adams DH. Immune interactions in hepatic fibrosis. Clin Liver Dis 2008;12:861-882.

6. Genescà J, Buti M. Cytokines and liver cirrhosis. Med Clin 2004; 122:457-458.

7. Hellerbrand C. Molecular targets for antifibrotic therapy in liver disease: using magic bullets for crossfire rather than a one-sided shotgun attack. Gut 2014;63:1039-1041.

8. Sancho-Bru P, Bataller R, Colmenero J, et al. Norepinephrine induces calcium spikes and proinflammatory actions in human hepatic stellate cells. Am J Physiol Gastrointest Liver Physiol 2006;291:G877-G884.

9. Oben JA, Roskams T, Yang S, et al. Hepatic fibrogenesis requires sympathetic neurotransmitters. Gut 2004;53:438-445.

10. Oben JA, Roskams T, Yang S, et al. Sympathetic nervous system inhibition increases hepatic progenitors and reduces liver injury. Hepatology 2003;38:664-673.

11. Dubuisson L, Desmouliere A, Decourt B, et al. Inhibition of rat liver fibrogenesis through noradrenergic antagonism. Hepatology 2002;35:325-331.
12. Tripathi D, Hayes PC. The role of carvedilol in the management of portal hypertension. Eur J Gastroenterol Hepatol 2010;22:905911.

13. Dandona P, Ghanim H, Brooks DP. Antioxidant activity of carvedilol in cardiovascular disease. J Hypertens 2007;25:731-741.

14. Hamdy N, El-Demerdash E. New therapeutic aspect for carvedilol: antifibrotic effects of carvedilol in chronic carbon tetrachlorideinduced liver damage. Toxicol Appl Pharmacol 2012;261:292299.

15. Lepor H. The evolution of alpha-blockers for the treatment of benign prostatic hyperplasia. Rev Urol 2006;8 Suppl 4:S3-S9.

16. Gressner AM, Weiskirchen R. Modern pathogenetic concepts of liver fibrosis suggest stellate cells and TGF-beta as major players and therapeutic targets. J Cell Mol Med 2006;10:76-99.

17. Junqueira LC, Cossermelli W, Brentani R. Differential staining of collagens type I, II and III by Sirius Red and polarization microscopy. Arch Histol Jpn 1978;41:267-274.

18. Roskams T, Cassiman D, De Vos R, Libbrecht L. Neuroregulation of the neuroendocrine compartment of the liver. Anat Rec A Discov Mol Cell Evol Biol 2004;280:910-923.

19. Motoyama H, Komiya T, Thuy le TT, et al. Cytoglobin is expressed in hepatic stellate cells, but not in myofibroblasts, in normal and fibrotic human liver. Lab Invest 2014;94:192-207.

20. Komeda K, Takai S, Jin D, et al. Chymase inhibition attenuates tetrachloride-induced liver fibrosis in hamsters. Hepatol Res 2010;40: 832-840.

21. Popper H. Pathologic aspects of cirrhosis: a review. Am J Pathol 1977;87:228-264.

22. Santra A, Chowdhury A, Ghosh A, Mazumder DN. Development of an animal model of hepatic fibrosis by excretory-secretory antigen of Ascaris suum. Indian J Gastroenterol 2000;19:119-121.

23. Gallego-Delgado J, Lazaro A, Gomez-Garre D, et al. Long-term organ protection by doxazosin and/or quinapril as antihypertensive therapy. J Nephrol 2006;19:588-598.

24. Perlini S, Palladini G, Ferrero I, et al. Sympathectomy or doxazosin, but not propranolol, blunt myocardial interstitial fibrosis in pressure-overload hypertrophy. Hypertension 2005;46:1213-1218.

25. Pawluczyk IZ, Patel SR, Harris KP. The role of the alpha-1 adrenoceptor in modulating human mesangial cell matrix production. Nephrol Dial Transplant 2006;21:2417-2424.

26. Shi MN, Huang YH, Zheng WD, Zhang LJ, Chen ZX, Wang XZ. Relationship between transforming growth factor beta 1 and anti-fibrotic effect of interleukin-10. World J Gastroenterol 2006;12:2357-2362.

27. Aldaba-Muruato LR, Moreno MG, Shibayama M, Tsutsumi V, Muriel P. Allopurinol reverses liver damage induced by chronic carbon tetrachloride treatment by decreasing oxidative stress, TGF-beta production and NF-kappaB nuclear translocation. Pharmacology 2013; 92:138-149. 